$R M x A C, \mathbf{5 3}, 134-136(2021)$

(c) 2021: Instituto de Astronomía, Universidad Nacional Autónoma de México

https://doi.org/10.22201/ia.14052059p.2021.53.26

\title{
SPINAR MODEL FOR BINARY NEUTRON STAR MERGER
}

\author{
A. R. Chasovnikov ${ }^{1}$, V. M. Lipunov ${ }^{1}$, and E. S. Gorbovskoy ${ }^{1}$
}

RESUMEN

Consideramos la fusión de las estrellas de neutrones desde el punto de vista del modelo de spinar. Presentamos cálculos de la luminosidad máxima de la fusión de estrellas de neutrones, tanto la luminosidad total como la luminosidad en el rango óptico. La posibilidad de observar tales explosiones de rayos gamma utilizando el sistema de telescopios robóticos MASTER también se discute.

\section{ABSTRACT}

We consider the neutron stars mergers from the point of view of the spinar model. We present calculations of the maximum luminosity of merging neutron stars, both total and in optical ranges. The possibility of observing such gamma-ray bursts using the MASTER system of robotic telescopes is also discussed.

Key Words: gravitational waves - stars: neutron

\section{INTRODUCTION}

In 2017 , gravitational waves from the collision of two neutron stars were observed for the first time (Abbott et al. 2017). Such mergers are an important phenomenon in the Universe, which can be observed both with the help of gravitational wave detectors and with the help of electromagnetic radiation receivers. And if the kilonova, which appears a few hours after the merger, is visible long enough, then the flash that occurs directly during the merger lasts about $2 \mathrm{~s}$, alike any short gamma-ray burst.

The experience of registering the first merger involving neutron stars shows that the phenomenon itself can be detected by gravitational detectors a few minutes before the merger itself. On the other hand, modern robotic telescopes of the global MASTER network are constantly following gamma-ray bursts during the first minute after receiving the alert (Lipunov et al. 2010). This means that if the GW detectors are able to give an alert a few minutes before the merger, there is a real opportunity to observe by astronomical telescopes the fusion phenomenon at the stage preceding the merger itself.

The MASTER robotic telescope system is constantly involved in the search for optical transients associated with gravitational wave events. The MASTER-IAC telescope was one of six telescopes to independently detect the kilonova GW 170817 (Lipunov et al. 2017).

We used the spinar model to calculate the evolution of the source. A spinar is a quasi-equilibrium fast-rotating compact object. This equilibrium is

\footnotetext{
${ }^{1}$ Moscow State University, Moscow, Universitetsky pr. 13, Moskow 119992, Russia.
}

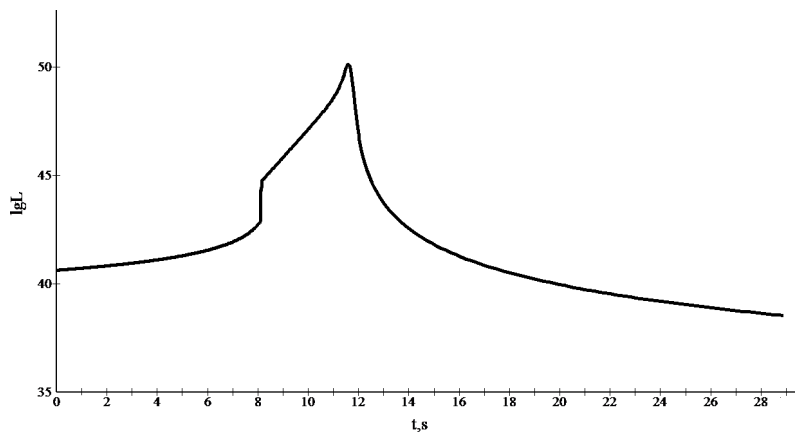

Fig. 1. Total luminosity. $B=10^{13} \mathrm{G}, \mathrm{M}=4 \mathrm{Mo}$

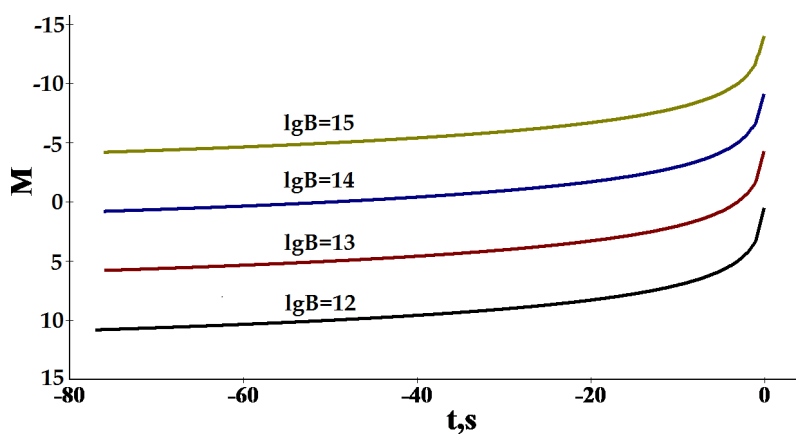

Fig. 2. Absolute magnitude. $\mathrm{M}=4 \mathrm{Mo}$

achieved by the action of two opposing forces - centrifugal and gravitational. The idea of spinar was developed a long time ago and was used to explain various phenomena, such as gamma-ray bursts (Lipunov et al. 1995; Lipunova et al. 1997, 1998, 2009).

In these articles, three-dimensional calculations are performed taking into account the effects caused by the presence of a magnetic field (Sun et al. 2019; Ruiz et al. 2019). 


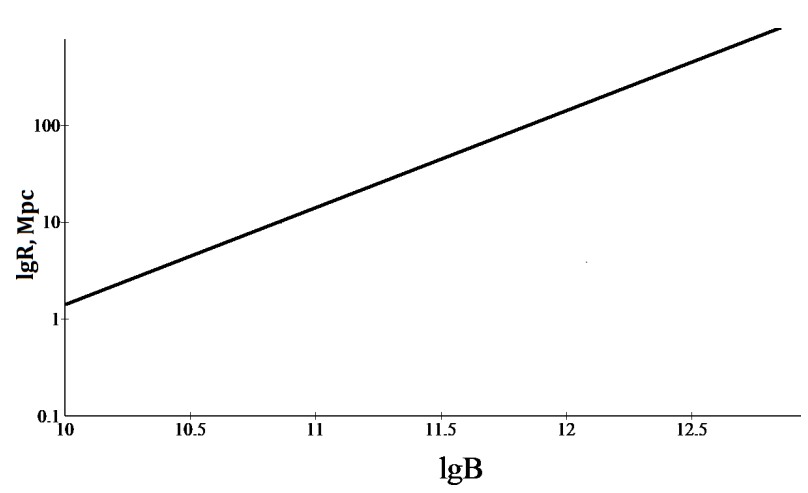

Fig. 3. The distance at which the MASTER system can see the spinar.

Our calculations contain two stages of the neutron star merger. The first is the convergence of two neutron stars, caused mainly by the radiation of gravitational waves. The second is the evolution of the spinar formed after the merger. The further fate of this object is determined, first of all, by the ratio of its mass and the Oppenheimer-Volkov limit.

\section{MODEL}

So, in our model, the following effects were taken into account:

1. Nuclear pressure.

2. The Lense - Thirring Effect.

3. The disappearance of the magnetic field during the collapse.

4. The loss of angular momentum caused by magnetic torque.

\section{The dynamo mechanism.}

We have considered two cases. The first, when the total mass is less than the Oppenheimer-Volkov limit, the second, when the total mass exceeds this limit. Recall that we considered the OppenheimerVolkov limit to be 2.3 times the mass of the Sun.

We use a very simple qualitative equation of state, but it takes into account the main factors that affect the spinar:

$$
\frac{d^{2} R}{d t}=F_{g}+F_{n}+F_{c}+F_{d i s s}
$$

where $F_{g}$ - gravitational force (taking into account the effects of GRT)(Mukhopadhyay 2002), $F_{n}$ - nuclear pressure, $F_{c}$ - centrifugal force and $F_{\text {diss }}$ - dissipative force. Of course, we are talking about a few specific forces.

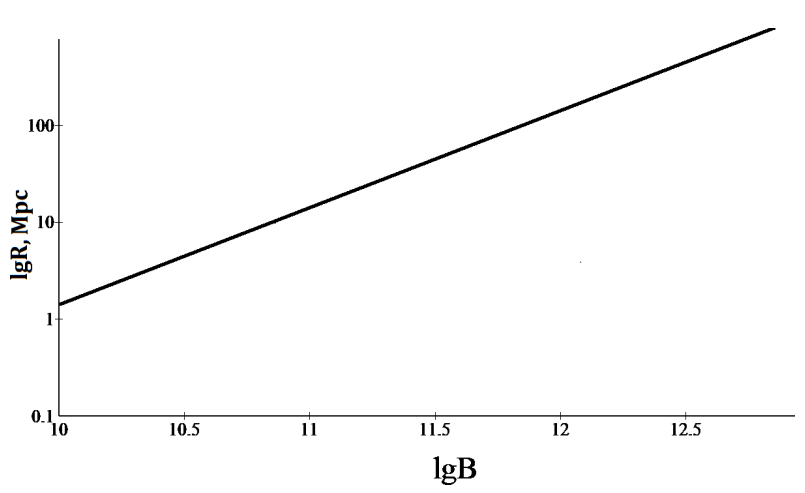

Fig. 4. The distance at which wide-field cameras can see the spinar

Quadrupole and dipole formulas are used to calculate luminosity.

$$
\begin{gathered}
L=\frac{\dddot{D}^{2}}{180 c^{5}} \\
L=\frac{\mu^{2} \omega}{R^{3}}
\end{gathered}
$$

Here

$$
D=\omega^{3} a \mu
$$

$\mu$ - the dipole magnetic moment. $M$ - the total mass of the components, $\omega$ - the angular velocity of spinar rotation.

Our calculations, unfortunately, give only the integral luminosity. Naturally, the practical interest is the flow of radiation in a particular range, especially in the optical (since optical radiation allows you to localize the GW event). We used the pulsar spectrum in the crab nebula to find the fraction of radiation in each band (Razdan 2015).

\section{RESULTS}

So, we have considered two cases. The first, when the total mass is less than the Oppenheimer-Volkov limit, the second, when the total mass exceeds this limit. We have calculated the total luminosity, but it is emitted mainly in the jet. Figure one shows an example of this calculation for merging neutron stars with a total mass of 4 times the mass of the Sun and a magnetic field of $10^{13} \mathrm{G}$. However, until the merger, we can assume that the jet has not yet been formed, so the energy is radiated isotropically. Below are the dependencies of the absolute magnitude of the star with time.

Figures 3 and 4 show the distances at which MASTER telescopes and ultra-wide field cameras 
are able to see radiation from neutron star mergers, depending on the magnetic field.

Therefore we have the following preliminary results: 1) MASTER telescopes can observe such gamma-ray bursts if they occur at a distance closer than 100-150 Mpc; 2) at extremely large magnetic fields, they can be observed at larger distances.

\section{REFERENCES}

Abbott, B. P., Abbott, R., Abbott, T. D., et al. 2017, ApJ 848, 12

Brown, E. F., Cumming, A., Fattoyev, F. J., et al. 2018, PhRvL, 120, 2701

Lipunov, V. M., Postnov, K. A., Prokhorov, M. E., Panchenko, I. E., \& Jorgensen, H. E. 1995, ApJ, 454, 593

Lipunov, V. M. \& Panchenko, I. E. 1996, A\&A 312, 937
Lipunov, V. M \& Gorbovskoy, E. S. 2007, ApJ, 665, 97

Lipunov, V. M., Kornilov, V., Gorbovskoy, E., et al. 2010, AdAst, 2010, 30

Lipunov, V. M., Gorbovskoy, E., Kornilov, V. G., et al. 2017, ApJ, 850, 1

Lipunov, V. M., Kornilov, V., Gorbovskoy, E., et al. 2018, NewA, 63, 48

Lipunova, G. V. 1997, AstL, 23, 84

Lipunova, G. V. \& Lipunov V. M. 1998, A\&A, 329, 29

Lipunova, G. V., Gorbovskoy, E. S., Bogomazov, A. I., \& Lipunov, V. M. 2009, MNRAS, 397, 1695

Mukhopadhyay, B. 2002, ApJ, 581, 427

Razdan, A. 2015, NewA, 37, 5

Ruiz, M., Tsokaros, A., Paschalidis, V., \& Shapiro, S. L. 2019, PhRvD, 99, 4032

Stratta, G., Dainotti, M. G., Dall'Osso, S., Hernandez, X., \& De Cesare, G. 2018, ApJ, 869, 155

Sun, L., Ruiz, M., \& Shapiro, S. L. 2019, PhRvD, 99, 4057 